\title{
All Ramsey numbers for brooms in graphs
}

\author{
Pei $\mathrm{Yu}$ \\ Department of Mathematics \\ Tong.ji University \\ Shanghai, China \\ yupeizjy@163.com
}

\author{
Yusheng Li \\ Department of Mathematics \\ Tongji University \\ Shanghai, China \\ li_yusheng@tongji.edu.cn
}

Submitted: Jul 17, 2015; Accepted: Aug 2, 2016; Published: Aug 19, 2016

Mathematics Subject Classifications: 05D10

\begin{abstract}
For $k, \ell \geqslant 1$, a broom $B_{k, \ell}$ is a tree on $n=k+\ell$ vertices obtained by connecting the central vertex of a star $K_{1, k}$ with an end-vertex of a path on $\ell-1$ vertices. As $B_{n-2,2}$ is a star and $B_{1, n-1}$ is a path, their Ramsey number have been determined among rarely known $R\left(T_{n}\right)$ of trees $T_{n}$ of order $n$. Erdős, Faudree, Rousseau and Schelp determined the value of $R\left(B_{k, \ell}\right)$ for $\ell \geqslant 2 k \geqslant 2$. We shall determine all other $R\left(B_{k, \ell}\right)$ in this paper, which says that, for fixed $n, R\left(B_{n-\ell, \ell}\right)$ decreases first on $1 \leqslant \ell \leqslant 2 n / 3$ from $2 n-2$ or $2 n-3$ to $\left\lceil\frac{4 n}{3}\right\rceil-1$, and then it increases on $2 n / 3<\ell \leqslant n$ from $\left\lceil\frac{4 n}{3}\right\rceil-1$ to $\left\lfloor\frac{3 n}{2}\right\rfloor-1$. Hence $R\left(B_{n-\ell, \ell}\right)$ may attain the maximum and minimum values of $R\left(T_{n}\right)$ as $\ell$ varies.
\end{abstract}

Keywords: Ramsey number; Tree; Broom

\section{Introduction}

Given a graph $G$, the Ramsey number $R(G)$ is the smallest integer $N$ such that every redblue coloring of the edges of $K_{N}$ contains a monochromatic $G$. Let $T_{n}$ be a tree of order $n$. Finding $R\left(T_{n}\right)$ for an arbitrary $T_{n}$ is a difficult unsolved problem in Ramsey theory. Most works focus on improving the known bounds, see [10]. Erdős and Sós conjectured that if a graph $G$ has average degree greater than $n-1$, then $G$ contains every tree of $n$ edges, which implies that $R\left(T_{n}\right) \leqslant 2 n-2$ for $n \geqslant 2$. A result of Erdős, Faudree, Rousseau and Schelp in [4] yields

$$
r\left(T_{n}\right) \geqslant\left\lceil\frac{4 n}{3}\right\rceil-1
$$

under (2) by minimizing the lower bound with $b=2 a$, and the lower bound can be attained by some brooms. For $k, \ell \geqslant 1$, a broom $B_{k, \ell}$ is a tree on $k+\ell$ vertices obtained by connecting the central vertex of a star $K_{1, k}$ with an end-vertex of a path on $\ell-1$ 
vertices. Thus $B_{k, 1}=K_{1, k}, B_{k, 2}=K_{1, k+1}$ and $B_{1, \ell}=P_{\ell+1}$, where $P_{\ell+1}$ is a path of order $\ell+1$. They obtained the following result.

Theorem 1. ([4]) Let $k$ and $\ell$ be integers with $\ell \geqslant 2 k \geqslant 2$ and $n=k+\ell$. Then

$$
R\left(B_{k, \ell}\right)=n+\left\lceil\frac{\ell}{2}\right\rceil-1
$$

Thus $R\left(B_{k, \ell}\right)=\left\lceil\frac{4 n}{3}\right\rceil-1$ for $\ell \in\{2 k, 2 k+1,2 k+2\}$ and $n=k+l$, which attain the lower bound in (1). In this paper, we shall determine the values of $R\left(B_{k, \ell}\right)$ for $1 \leqslant \ell \leqslant 2 k-1$.

Note that when $k$ is fixed and $\ell$ is sufficient large, $B_{k, \ell}$ is similar to a path $P_{n}$; when $\ell$ is fixed and $k$ is sufficient large, $B_{k, \ell}$ is similar to a star $K_{1, n-1}$. Among few known results of $R\left(T_{n}\right), R\left(P_{n}\right)$ and $R\left(K_{1, n-1}\right)$ have been determined completely. For $n \geqslant 2$, the exact value of $R\left(P_{n}\right)$ was determined in [7] as

$$
R\left(P_{n}\right)=\left\lfloor\frac{3 n}{2}\right\rfloor-1
$$

and $R\left(K_{1, n-1}\right)$ was determined in [3] as

$$
R\left(K_{1, n-1}\right)= \begin{cases}2 n-3 & \text { if } n \text { is odd } \\ 2 n-2 & \text { otherwise }\end{cases}
$$

As $B_{1, \ell}=P_{\ell+1}, B_{k, 1}=K_{1, k}$ and $B_{k, 2}=K_{1, k+1}$, their Ramsey numbers can be determined by the above results. It was proved that $R\left(B_{k, 3}\right)=R\left(K_{1, k+1}\right)$ in [2]. Thus we shall consider the case $\ell \geqslant 4$ and $k \geqslant 2$.

Theorem 2. Let $k$ and $\ell$ be integers with $k \geqslant 2$ and $n=k+\ell$. Then

$$
R\left(B_{k, \ell}\right)=\left\{\begin{array}{c}
n+\left\lceil\frac{\ell}{2}\right\rceil-1 \quad \text { if } \ell \geqslant 2 k-1 \\
2 n-2\left\lceil\frac{\ell}{2}\right\rceil-1 \quad \text { if } 4 \leqslant \ell \leqslant 2 k-2 .
\end{array}\right.
$$

Remark. Roughly speaking, for fixed $n, R\left(B_{n-\ell, \ell}\right)$ decreases first on $2 \leqslant \ell \leqslant \frac{2 n-1}{3}$ from $2 n-2$ or $2 n-3$ to $\left\lceil\frac{4 n}{3}\right\rceil-1$, and then increases on $\frac{2 n-1}{3}<\ell \leqslant n$ from $\left\lceil\frac{4 n}{3}\right\rceil-1$ to $\left\lfloor\frac{3 n}{2}\right\rfloor-1$. Hence $R\left(B_{n-\ell, \ell}\right)$ may attain the maximum and minimum values of $R\left(T_{n}\right)$ when $\ell$ varies, as it is believed that $R\left(K_{1, n-1}\right)$ is the maximum value of $R\left(T_{n}\right)$.

\section{Proofs}

For any red-blue edge-coloring of $K_{N}$, denote $R$ and $B$ be the induced red and blue subgraph, respectively, and $N_{R}(x)$ and $N_{B}(x)$ be the red neighborhood and blue neighborhood of $x$, respectively. Let $N_{R}[x]=N_{R}(x) \cup\{x\}, N_{B}[x]=N_{B}(x) \cup\{x\}, \operatorname{deg}_{R}(x)=\left|N_{R}(x)\right|$, and $\operatorname{deg}_{B}=\left|N_{B}(x)\right|$. For a graph $G$ and disjoint subset $A$ and $D$, denote by $G(A)$ the subgraph of $G$ induced by $A$, and $G(A, D)$ the bipartite subgraph of $G$ induced by $A$ and $D$. If $G$ is the red-blue edge-colored $K_{N}$, we write $G_{R}(A)=G(A) \cap R$ and $G_{R}(A, D)=G(A, D) \cap R$. Notation not specifically mentioned will follow from [1]. We do not distinguish the vertex set and the graph when there is no danger of confusion. 
Consider a tree $T_{n}$ as a bipartite graph with two parts of size $a$ and $b$, respectively, where $a \leqslant b, a+b=n$. Observing that a red-blue edge-colored $K_{2 a+b-2}$ with $R=$ $K_{a-1} \cup K_{a+b-1}$ contains no monochromatic $T_{n}$, and a red-blue edge-colored $K_{2 b-2}$ with $R=K_{b-1} \cup K_{b-1}$ contains no monochromatic $T_{n}$. We see that

$$
R\left(T_{n}\right) \geqslant \max \{2 a+b-1,2 b-1\},
$$

where $a$ and $b$ are determined by $T_{n}$.

Note that $B_{k, \ell}$ is a bipartite graphs on parts of sizes $a=\left\lceil\frac{\ell}{2}\right\rceil$ and $b=k+\left\lfloor\frac{\ell}{2}\right\rfloor$, then

$$
R\left(B_{k, \ell}\right) \geqslant \max \left\{k+\left\lceil\frac{3 \ell}{2}\right\rceil-1,2 k+2\left\lfloor\frac{\ell}{2}\right\rfloor-1\right\}
$$

We shall prove the cases $\ell=2 k-1$ and $\ell=4$ in Theorem 2 via the following two lemmas.

Lemma 3. Let $k \geqslant 2$ be an integer. Then

$$
R\left(B_{k, 2 k-1}\right)=4 k-2 .
$$

Lemma 4 . Let $k \geqslant 2$ be an integer. Then

$$
R\left(B_{k, 4}\right)=2 k+3
$$

In order to prove Lemma 3, we need two results from [9] and [6], respectively.

Lemma 5. ([9]) Let $G(A, D)$ be a bipartite graph on parts $A$ and $D$ with $|A|=k$ and $|D|=2 k-2$ such that

$$
\min \{d(x): x \in A\} \geqslant k .
$$

Then $G(A, D)$ contains a cycle $C_{2 k}$.

Lemma 6. ([6]) $R\left(C_{2 k}\right)=3 k-1$ for integer $k \geqslant 3$

Proof of Lemma 3. It is easy to see that $R\left(B_{2,3}\right)=6$, we assume that $k \geqslant 3$. As the lower bound (3) implies $R\left(B_{k, 2 k-1}\right) \geqslant 4 k-2$, it suffices to show the opposite inequality.

Let $G$ be a red-blue edge-colored $K_{4 k-2}$. We shall show that $G$ contains a monochromatic $B_{k, 2 k-1}$. By lemma $6, G$ contains a monochromatic cycle $C_{2 k}$. Without loss of generality, we assume that this $C_{2 k}$ is blue and denote it by $C_{2 k}^{(B)}$. Let $D=G \backslash C_{2 k}^{(B)}$. Then $|D|=2 k-2$. If there exists a vertex $x \in C_{2 k}^{(B)}$ such that $\left|N_{B}(x) \cap D\right| \geqslant k-1$, then $G$ contains a blue $B_{k, 2 k-1}$. We then assume that $\left|N_{B}(x) \cap D\right| \leqslant k-2$ for each $x \in C_{2 k}^{(B)}$. The fact that

$$
\left|N_{B}(x) \cap D\right|+\left|N_{R}(x) \cap D\right|=|D|=2 k-2
$$

implies that $\left|N_{R}(x) \cap D\right| \geqslant k$ for each $x \in C_{2 k}^{(B)}$, hence the number of red edges between $C_{2 k}^{(B)}$ and $D$ is at least $2 k^{2}$. So there exists a vertex $u \in D$ such that

$$
\left|N_{R}(u) \cap C_{2 k}^{(B)}\right| \geqslant \frac{2 k^{2}}{|D|}=\frac{2 k^{2}}{2 k-2} \geqslant k+1 .
$$


Let $\left\{u_{0}, u_{1}, \ldots, u_{k}\right\} \subseteq N_{R}(u) \cap C_{2 k}^{(B)}$, and $A=C_{2 k}^{(B)} \backslash\left\{u_{1}, u_{2}, \ldots, u_{k}\right\}$. Then $|A|=k$. Consider the bipartite graph $G_{R}(A, D)$. By Lemma 5 , there is a red cycle $C_{2 k}$ between $A$ and $D$. Denote by $C_{2 k}^{(R)}$ the red $C_{2 k}$. Since $C_{2 k}^{(R)}$ contains $A$ hence $u_{0}$, so the graph on $\left\{u_{1}, u_{2}, \ldots, u_{k}\right\}, u, u_{0}, C_{2 k}^{(R)}$ induced by red edges contains a red $B_{k, 2 k-1}$.

This completes the proof of Lemma 3.

Proof of Lemma 4. The lower bound (3) implies $R\left(B_{k, 4}\right) \geqslant 2 k+3$, and we shall show $R\left(B_{k, 4}\right) \leqslant 2 k+3$. Let $G$ be a red-blue edge-colored $K_{2 k+3}$. Assume that $G$ contains no monochromatic $B_{k, 4}$.

If $k=2,2 k+3=7$. As $R\left(P_{5}\right)=6<7$, we suppose that $G$ contain a red $P_{5}$. Label the vertices of the path in order as $\left\{x_{1}, x_{2}, \ldots, x_{5}\right\}$, denote another two vertices as $y_{1}, y_{2}$. Since there is no red $B_{2,4}$, all the edges between $\left\{x_{2}, x_{4}\right\}$ and $\left\{y_{1}, y_{2}\right\}$ are red. If there is red edge between $\left\{x_{1}, x_{5}\right\}$ and $\left\{y_{1}, y_{2}\right\}$, say edge $x_{5} y_{2}$ is red. Then edges $x_{5} y_{1}, x_{3} y_{1}, x_{3} y_{2}$ are blue. Now $\left\{x_{2}, x_{3}\right\}, y_{2}, x_{4}, y_{1}, x_{5}$ contain a blue $B_{2,4}$, a contradiction. If all the edges between $\left\{x_{1}, x_{5}\right\}$ and $\left\{y_{1}, y_{2}\right\}$ are blue, then $\left\{x_{1}, x_{2}\right\}, y_{2}, x_{4}, y_{1}, x_{5}$ contain a blue $B_{2,4}$, a contradiction.

Now we consider the case $k \geqslant 3$. As $R\left(K_{1, k+1}\right)<2 k+3$, we suppose that there is a blue star $K_{1, k+1}$, which is denoted by $K_{1, k+1}^{(B)}$. Let $x$ be the center of $K_{1, k+1}^{(B)}, A=K_{1, k+1}^{(B)} \backslash\{x\}$ and $D=G \backslash K_{1, k+1}^{(B)}$. Then $|A|=|D|=k+1$.

Claim. $D$ induces a red $K_{k+1}$.

Proof. Suppose to the contrary, there is a blue edge $u v$ in $D$. Since $G$ contains no blue $B_{k, 4}$, the edges between $\{u, v\}$ and $A$ are all red, and thus all the edges between $\{u, v\}$ and $D \backslash\{u, v\}$ are blue from the assumption that $G$ contains no red $B_{k, 4}$. Now consider the blue edges between $\{u, v\}$ and $A$. With a similar analysis, we get that $D$ induces a blue $K_{k+1}$ and all edges between $D$ and $A$ are red.

Now, consider the adjacency between $x$ and a vertex of $D$, say $x u$, no matter what the color of $x u$ is, we have a monochromatic $B_{k, 4}$, leading to a contradiction and the claim is proved.

Now $D$ is a red $K_{k+1}$. If there exists a red edge $x w$ with $w \in D$, then $D \cup\{x\}$ induces a red $K_{1, k+1}$ with center $w$. As $A=V(G) \backslash(D \cup\{x\})$, a similar analysis for the above claim tells us that $A$ is a blue $K_{k+1}$. If the number of blue edges between $A$ and $D$ is at least $k+2$, then there exists a vertex $y \in A$ such that $\left|N_{B}(y) \cap D\right| \geqslant 2$. Now choose two vertices $\left\{y_{1}, y_{2}\right\} \subseteq N_{B}(y) \cap D$ and two vertices $\left\{a_{1}, a_{2}\right\} \subseteq A \backslash y$, then $\left(A \backslash\left\{a_{1}, a_{2}, y\right\}\right) \cup\left\{y_{1}, y_{2}\right\}, y, a_{1}, a_{2}, x$ contains a blue $B_{k, 4}$, a contradiction. Thus assume to the contrary, there exists a vertex $z \in D$ such that $\left|N_{R}(z) \cap A\right| \geqslant \frac{(k+1)^{2}-(k+1)}{|D|}=k \geqslant 2$. If $w, z$ are the same vertex, we can choose two vertices $\left\{z_{1}, z_{2}\right\} \subseteq N_{R}(z) \cap A$ and three vertices $\left\{d_{1}, d_{2}, d_{3}\right\} \subseteq D \backslash z$ for $|D|=k+1 \geqslant 4$. Then $\left(D \backslash\left\{d_{1}, d_{2}, d_{3}, z\right\}\right) \cup\left\{z_{1}, z_{2}, x\right\}, z, d_{1}, d_{2}, d_{3}$ contain a red $B_{k, 4}$. If $w, z$ are different, choose a vertex $d_{1} \in D \backslash\{z, w\}$, then $(D \backslash$ $\left.\left\{z, w, d_{1}\right\}\right) \cup\left\{z_{1}, z_{2}\right\}, z, d_{1}, w, x$ contain a red $B_{k, 4}$, a contradiction.

Finally, assume that $x$ is adjacent to $D$ completely blue. Choose any set $F \subseteq A \cup D$ such that $|F|=k+1$ and denote $M=V(G) \backslash(F \cup x)$. A similar analysis for the claim says that $M$ is a red $K_{k+1}$. The choice of $F$ tells us that $A \cup D$ is a red $K_{2 k+2}$, hence $\mathrm{G}$ 
contains a red $B_{k, 4}$, which is a contradiction too.

This completes the proof of Lemma 4.

Lemma 7. For integers $k, \ell, N$ with $5 \leqslant \ell \leqslant 2 k-2$ and $N \geqslant 2 k+2\left\lfloor\frac{\ell}{2}\right\rfloor-1$, let the edges of $K_{N}$ be colored by two colors $i \equiv 0,1(\bmod 2)$. Suppose $i$ is a color and $x$ is a vertex such that

$$
\operatorname{deg}_{i}(x)=\max _{v} \max \left\{\operatorname{deg}_{i}(v), \operatorname{deg}_{i+1}(v)\right\} .
$$

If there exist vertices $y, z \subseteq N_{i+1}(x)$, not necessarily distinct, satisfying

1. $\left|N_{i+1}(y) \cap N_{i}(x)\right| \geqslant k$, and

2. $\operatorname{deg}_{i+1}(z) \geqslant N-\ell$

then $G$ contains a monochromatic $B_{k, \ell}$.

Proof of Lemma \%. Since $\left|N_{i+1}(y) \cap N_{i}(x)\right| \geqslant k$, we can choose a subset $A$ in $N_{i+1}(y) \cap$ $N_{i}(x)$ such that $|A|=k$. Let $H=G \backslash A$, then $|H|=N-k$. Since $R\left(C_{2 t}\right)=3 t-1$ for $t \geqslant 3, H$ contains a monochromatic $C_{2 t}$ in color $j$, denoted by $C_{2 t}^{(j)}$, where

$$
2 t \geqslant 2\left\lfloor\frac{N-k+1}{3}\right\rfloor \geqslant 2\left\lfloor\frac{k+2\lfloor\ell / 2\rfloor}{3}\right\rfloor \geqslant \ell
$$

for $5 \leqslant \ell \leqslant 2 k-2$. The choice of vertex $x \operatorname{implies} \operatorname{deg}_{i}(x) \geqslant \operatorname{deg}_{i+1}(z) \geqslant N-\ell$, and thus

$$
\left|N_{i}[x] \backslash A\right|+\left|C_{2 t}^{(j)}\right| \geqslant|H|+1, \quad\left|N_{i+1}[z] \backslash A\right|+\left|C_{2 t}^{(j)}\right| \geqslant|H|+1,
$$

which implies that both $N_{i}[x] \backslash A$ and $N_{i+1}[z] \backslash A$ contain a vertex of $C_{2 t}^{(j)}$.

Case 1. $y=z$. If $j=i$, namely, $C_{2 t}^{(j)}=C_{2 t}^{(i)}$ is in color $i$, there is a monochromatic $B_{k, \ell}$ in color $i$ in $A \cup\{x\} \cup C_{2 t}^{(i)}$, and otherwise $j=i+1$, there exists a monochromatic $B_{k, \ell}$ in color $i+1$ in $A \cup\{y\} \cup C_{2 t}^{(i+1)}$.

Case 2. $y \neq z$. Similarly, we can find a monochromatic $B_{k, \ell}$ either in $A \cup\{x\} \cup C_{2 t}^{(j)}$ or in $A \cup\{y, x, z\} \cup C_{2 k}^{(j)}$.

This completes the proof of Lemma 7 .

The next two lemmas are results about the extremal edges in graph that contains no path $P_{t}$.

Lemma 8. ([5]) Let $t \geqslant 2$ be an integer, and $G$ a graph of order $N$ that contains no $P_{t}$. Let $e(G)$ be the number of edges of $G$, then $e(G) \leqslant \frac{(t-2) N}{2}$.

Lemma 9. ([8]) Let $G\left(X_{B}, X_{R}\right)$ be a bipartite graph on parts $X_{B}$ and $X_{R}$ with $\left|X_{R}\right| \leqslant$ $\left|X_{B}\right|$. If $G\left(X_{B}, X_{R}\right)$ contains no $P_{2 t}$ with $2(t-1) \leqslant\left|X_{R}\right|$, then

$$
e\left(G\left(X_{B}, X_{R}\right)\right) \leqslant(t-1)\left[\left|X_{B}\right|+\left|X_{R}\right|-2(t-1)\right] .
$$


Proof of Theorem 2. We may assume that $5 \leqslant \ell \leqslant 2 k-2$ from Theorem 1 , Lemma 3 and Lemma 4.

Set $N=2 n-2\left\lceil\frac{\ell}{2}\right\rceil-1=2 k+2\left\lfloor\frac{\ell}{2}\right\rfloor-1$. Let $G$ be a red-blue edge-colored $K_{N}$, and let $R$ and $B$ be the induced red and blue subgraph, respectively. Without loss of generality, we may assume that the maximal monochromatic degree of $G$ is the maximum blue degree and $x$ is a vertex such that

$$
\operatorname{deg}_{B}(x)=\max _{v} \max \left\{\operatorname{deg}_{B}(v), \operatorname{deg}_{R}(v)\right\} .
$$

To simplify the notation, we write $X_{B}=N_{B}(x), X_{R}=N_{R}(x)$ and

$$
t=\ell+k-\left|X_{B}\right|-1 \text {. }
$$

The choice of $x$ implies $N_{B}(u) \cap X_{B} \neq \emptyset$ for each vertex $u \in X_{R}$ as otherwise $N_{B}[x] \subseteq$ $N_{R}(u)$ and thus $\operatorname{deg}_{R}(u)>\operatorname{deg}_{B}(x)$, which is impossible. We shall separate the proof into three cases depending on $\left|X_{B}\right|$.

Case 1. $\left|X_{B}\right|<k+\ell-1$, and either $G\left(X_{R}\right)$ contains a blue $P_{t}$, denoted by $P_{t}^{(B)}$, or $G\left(X_{B}, X_{R}\right)$ contains a blue $P_{2 t}$, denoted $P_{2 t}^{(B)}$.

In $G\left(X_{B} \cup X_{R}\right)$, let $P^{(B)}$ be the longest blue path extended from $P_{t}^{(B)}$ such that one of its end-vertices is in $X_{B}$ if $G\left(X_{R}\right)$ contains a blue $P_{t}$, or that from $P_{2 t}^{(B)}$ otherwise. If $\left|P^{(B)}\right| \geqslant \ell-1$, then there exists a blue $B_{k, \ell}$. Thus we assume that $\left|P^{(B)}\right| \leqslant \ell-2$, then $P^{(B)}$ fails to contain at least $\left|X_{B}\right|-(\ell-2-t)=k+1$ vertices of $X_{B}$. Let $y$ be the other end-vertex of $P^{(B)}$. Then $\left|N_{R}(y) \cap X_{B}\right| \geqslant k+1$. The maximality of $\left|P^{(B)}\right|$ implies $\left|N_{R}(y)\right| \geqslant N-1-(\ell-2)=N-\ell+1$, which and Lemma 7 imply that $G$ contains a monochromatic $B_{k, \ell}$.

Case 2. $\left|X_{B}\right| \geqslant k+\ell-1$.

Let $P^{(B)}$ be the longest blue path in $G\left(X_{B} \cup X_{R}\right)$ that has an end-vertex in $X_{B}$. A similar analysis in Case 1 implies that $G$ contains a monochromatic $B_{k, \ell}$.

Case 3. $\left|X_{B}\right|<k+\ell-1$, and neither $G\left(X_{R}\right)$ contains a blue $P_{t}$ nor $G\left(X_{B}, X_{R}\right)$ contains a blue $P_{2 t}$.

As $t=\ell+k-\left|X_{B}\right|-1 \geqslant 2$, then $\left|X_{B}\right| \leqslant \ell+k-3$ and $\left|X_{R}\right|=N-1-\left|X_{B}\right| \geqslant k$.

Since $G\left(X_{R}\right)$ contains no blue $P_{t}$, Lemma 8 implies $e\left(G_{B}\left(X_{R}\right)\right) \leqslant(t-2)\left|X_{R}\right| / 2$. The choice of $x$ implies that the $\min \left\{\operatorname{deg}_{R}(v), \operatorname{deg}_{B}(v)\right\} \geqslant\left|X_{R}\right|$ for each vertex $v$ of $G$, and thus

$$
e\left(G_{B}\left(X_{B}, X_{R}\right)\right) \geqslant\left|X_{R}\right| \cdot\left|X_{R}\right|-(t-2)\left|X_{R}\right|=\left(\left|X_{R}\right|-t+2\right)\left|X_{R}\right| .
$$

Since $G\left(X_{B}, X_{R}\right)$ contains no blue $P_{2 t}$, Lemma 9 yields

$$
e\left(G_{B}\left(X_{B}, X_{R}\right)\right) \leqslant M_{B}
$$

where

$$
M_{B}=(t-1)\left[\left|X_{B}\right|+\left|X_{R}\right|-2(t-1)\right]
$$

Claim for Case 3. $G\left(X_{B}, X_{R}\right)$ has at most $\left|X_{R}\right|\left(\left|X_{B}-k\right|\right)-1$ blue edges. 
Proof. Suppose opposite, then

$$
e\left(G_{B}\left(X_{B}, X_{R}\right)\right) \geqslant\left|X_{R}\right| \cdot \max \left\{\left|X_{R}\right|-t+2,\left|X_{B}\right|-k\right\} \geqslant m_{B} .
$$

where

$$
m_{B}=k\left(\left|X_{R}\right|-t+2\right)+\left(\left|X_{R}\right|-k\right)\left(\left|X_{B}\right|-k\right) .
$$

Note that $M_{B}$ and $m_{B}$ are upper and lower bound of number of blue edges in $G\left(X_{B}, X_{R}\right)$, respectively, and thus $M_{B} \geqslant m_{B}$.

Case 3.1. $\ell$ is even. In this subcase, $\left|X_{B}\right|+\left|X_{R}\right|=2 k+\ell-2$ and

$$
\begin{aligned}
& t=\ell+k-\left|X_{B}\right|-1=\left|X_{R}\right|-k+1, \\
& m_{B}=k(k+1)+\left(\left|X_{R}\right|-k\right)\left(\left|X_{B}\right|-k\right), \\
& M_{B}=\left(\left|X_{R}\right|-k\right)\left[\left|X_{B}\right|+k-\left(\left|X_{R}\right|-k\right)\right]
\end{aligned}
$$

we have

$$
m_{B}-M_{B}=k^{2}-2 k\left(\left|X_{R}\right|-k\right)+\left(\left|X_{R}\right|-k\right)^{2}+k=\left(2 k-\left|X_{R}\right|\right)^{2}+k>0,
$$

which is a contradiction.

Case 3.2. $\ell$ is odd. In this subcase, $\left|X_{R}\right|+\left|X_{B}\right|=2 k+\ell-3$ and

$$
\begin{aligned}
& t=\ell+k-\left|X_{B}\right|-1=\left|X_{R}\right|-k+2, \\
& m_{B}=k^{2}+\left(\left|X_{R}\right|-k\right)\left(\left|X_{B}\right|-k\right), \\
& M_{B}=\left(\left|X_{R}\right|-k+1\right)\left[\left|X_{B}\right|+k-\left(\left|X_{R}\right|-k\right)-2\right] .
\end{aligned}
$$

We have

$$
\begin{aligned}
m_{B}-M_{B} & =\left(2 k-\left|X_{R}\right|\right)^{2}+3\left|X_{R}\right|-\left|X_{B}\right|-4 k+2 \\
& =\left(2 k-\left|X_{R}\right|\right)\left(2 k-\left|X_{R}\right|-4\right)+2 k-\ell+5 .
\end{aligned}
$$

For $\ell \leqslant 2 k-2$,is odd, we get $\left|X_{R}\right| \leqslant\left\lfloor\frac{N-1}{2}\right\rfloor \leqslant 2 k-3$. If $\left|X_{R}\right| \leqslant 2 k-4, m_{B}-M_{B} \geqslant$ $2 k-\ell+5>0$; if $\ell_{2}=2 k-3, m_{B}-M_{B}=2 k-\ell+2>0$, a contradiction, hence the claim holds.

We now have

$$
e\left(G_{R}\left(X_{R}\right)\right) \geqslant\left(\begin{array}{c}
\left|X_{R}\right| \\
2
\end{array}\right)-\frac{(t-2)\left|X_{R}\right|}{2} \geqslant \frac{(k-1)\left|X_{R}\right|}{2} .
$$

and

$$
e\left(\left(G_{R}\left(X_{B}, X_{R}\right)\right) \geqslant\left|X_{R}\right| \cdot\left|X_{B}\right|-\left[\left|X_{R}\right|\left(\left|X_{B}\right|-k\right)-1\right]=k\left|X_{R}\right|+1,\right.
$$

Recall $X_{R}=N_{R}(x)$, and thus

$$
\sum_{v \in X_{R}}\left|N_{R}(v)\right| \geqslant e\left(G_{R}\left(X_{B}, X_{R}\right)\right)+2 e\left(G_{R}\left(X_{R}\right)\right)+\left|X_{R}\right|=2 k\left|X_{R}\right|+1 .
$$

Therefore, there exist $y, z \subseteq X_{R}=N_{R}(x)$,not necessarily distinct, such that $\mid N_{R}(y) \cap$ $N_{B}(x) \mid \geqslant k+1$ and $\left|N_{R}(z)\right| \geqslant 2 k+1 \geqslant N-\ell$. Then, Lemma 7 implies that $G$ contains a monochromatic $B_{k, \ell}$.

This completes the proof of Theorem 2 . 


\section{Acknowledgements}

The authors are grateful to the editors and referees for their invaluable comments and suggestions, particularly, their careful reading and detailed marking on the manuscript.

\section{References}

[1] B. Bollobás. Modern Graph Theory. Springer-Verlag, New York, 1998.

[2] P. Bahls and T. Spencer. On the Ramsey numbers of trees with small diameter. Graphs Combin., 29:39-44, 2013.

[3] V. Chvátal and F. Harary. Generalized Ramsey theory for graphs II, Small diagonal numbers. Proc. Amer. Math. Soc., 32:389-394, 1972.

[4] P. Erdős, R. Faudree, C. Rousseau and R. Schelp. Ramsey numbers for brooms. Congr. Numer., 35:283-293, 1982.

[5] P. Erdös and T. Gallai. On maximal paths and circuits graphs. Acta Math. Acad. Sci. Hung., 10: 337-356, 1959.

[6] R. Faudree and R. Schelp. All Ramsey numbers for cycles in graphs. Discrete Math., $8: 35-52,1974$.

[7] L. Geréncser and A. Gyárfas. On Ramsey-type problems. Ann. Univ. Sci. Budapest, Eötvös Sect. Math., 10:167-170, 1967.

[8] A. Gyárfás, C. Rousseau and R. Schelp. An extremal problem for paths in bipartite graphs, J. Graph Theory., 8:83-95, 1984.

[9] B. Jackson. Cycles in bipartite graphs. J. Combin. Theory Ser. B., 30:332-342, 1981.

[10] S. Radziszowski. Small Ramsey numbers. Electronic J. Combin., \#DS1 1994. 